\title{
Integrating logic-based machine learning and virtual screening to discover new drugs
}

\author{
Christopher R Reynolds*, Michael JE Sternberg \\ From 7th German Conference on Chemoinformatics: 25 CIC-Workshop \\ Goslar, Germany. 6-8 November 2011
}

Investigational Novel Drug Discovery by Example (INDDEx ${ }^{\mathrm{TM}}$ ) is a technology developed to guide hit to lead discovery by learning rules from existing active compounds that link activity to chemical substructure. INDDEx is based on Inductive Logic Programming [1], which learns easily interpretable qualitative logic rules from active ligands that give an insight into chemistry, relate molecular substructure to activity, and can be used to guide the next steps of drug design chemistry. Support Vector Machines weight the rules to produce a quantitative model of structure-activity relationships. Whereas earlier testing $[2,3]$ was performed on single dataset examples, this talk presents the largest and fullest test of the method. The method was benchmarked on the Directory of Useful Decoys (DUD) datasets [4], using the same methodology described in the paper on the assessment of LASSO [5] and DOCK. For each of the DUD datasets, the known active ligands were mixed with all the decoy compounds in DUD, and the retrieval rates of INDDEx and DUD were measured when they were trained on 2, 4, and 8 of the known active ligands (Figure 2). Early retrieved compounds showed high topological differences to molecules used as training data, showing the strength of this method for scaffold hopping. This work was supported by a BBSRC case studentship with Equinox Pharma Ltd (http://www.equinoxpharma.com).
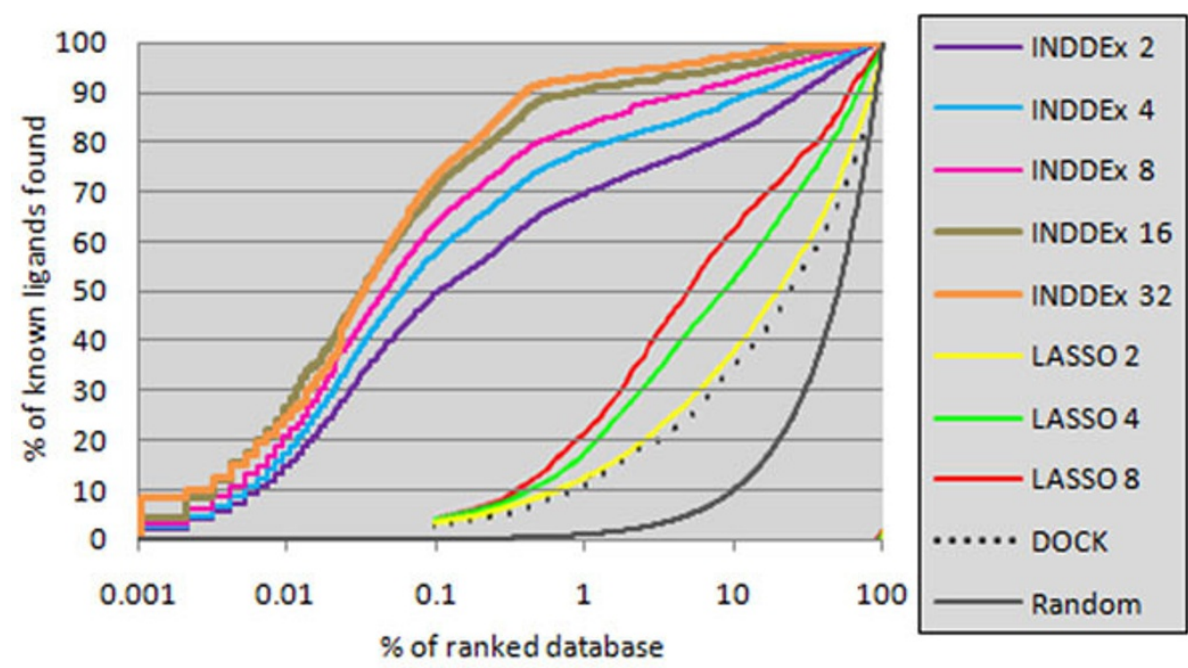

Figure 1 Recovery of actives in each of the DUD datasets from all decoys in the DUD, averaged across all 40 datasets.

\footnotetext{
* Correspondence: cr308@imperial.ac.uk Imperial College, London, N19 3LA, UK 


\section{References}

1. Muggleton SH: Inductive logic programming. New Gen Comp 1995, 13:245-286.

2. Amini A, et al: A Novel Logic-Based Approach for Quantitative Toxicology Prediction. J Chem Inf Model 2007, 47:998-1006.

3. Cannon EO, et al: Support vector inductive logic programming outperforms the naive Bayes classifier and inductive logic programming for the classification of bioactive chemical compounds. J Comput Aided Mol Des 2007, 21:269-280.

4. Huang N, et al: Benchmarking Sets for Molecular Docking. J Med Chem 2006, 49:6789-6801.

5. Reid D, et al: LASSO-ligand activity by surface similarity order: a new tool for ligand based virtual screening. J Comput Aided Mol Des 2008, 22:479-487.

doi:10.1186/1758-2946-4-S1-010

Cite this article as: Reynolds and Sternberg: Integrating logic-based machine learning and virtual screening to discover new drugs. Journal of Cheminformatics 2012 4(Suppl 1):O10.

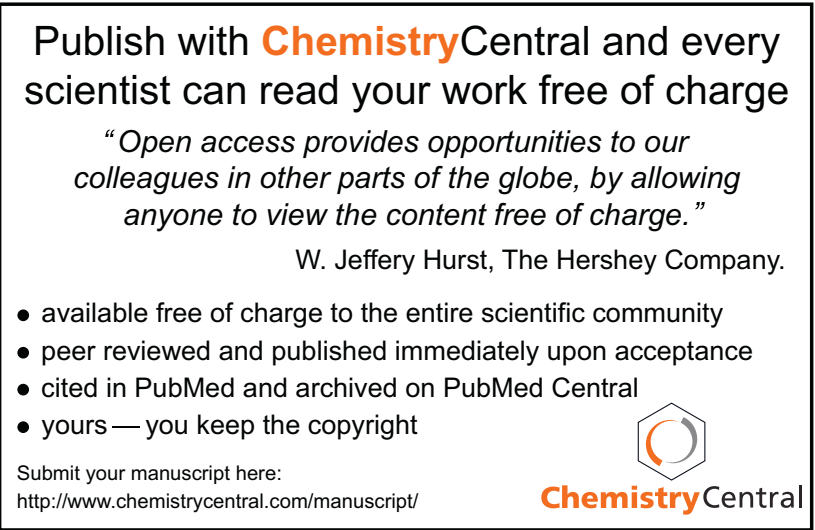

